\title{
High Resolution Micro-Pirani Pressure Sensor Gauge with Transient Response Processing.
}

\author{
O.Legendre ${ }^{*}$, H.Mathias ${ }^{*}$, J.Julliard ${ }^{+}$E.Martincic ${ }^{*}$, F.Mailly ${ }^{\mathrm{x}}$ \\ "Université Paris Sud XI/CNRS, IEF, F91405 Orsay, France, legendre.o@ gmail.com \\ ${ }^{+}$Department of Measurement, SUPÉLEC Plateau du Moulon, 91192 Gif-sur-Yvette Cedex, France \\ ${ }^{\mathrm{x}}$ Université Montpellier 2/CNRS, LIRMM, 161 rue Ada, 34392 Montpellier, France
}

\section{Summary}

A micro-Pirani pressure sensor which acts as a pressure dependent thermo-resistance gauge is traditionally exploited using a steady state resistance measurement. However any signal variation occurs over a constant voltage bias due to the initial resistance of the device which affects the sensor's sensitivity. Our work shows for the first time an experimental investigation of a microPirani gauge based on its dynamical behavior when heated by a current step. Such a processing does magnify the pressure dependence of the gauge's signal in eliminating the initial resistance influences on the measurement. Furthermore, a first order low pass filter step response identification of the experimental transient signal strongly reduces the thermal noise influence on the measurement. The heating step, the recording of the time dependent signal and its postprocessing can be easily achieved by a small-size controller. The proposed system provides a substantial enhancement of the micro-Pirani pressure sensor performance.

\section{Motivation}

Size scaling of pressure sensors is an active field of investigation. Applications like vacuum packaging hermetic control require small-size absolute pressure sensors. The Pirani devices, which act as a pressure dependent thermo-resistance for low pressure levels, are very good candidates for such applications. Micro-Pirani gauges can indeed easily be obtained using micro-machining techniques ${ }^{12}$ (see Fig. 1). However due to micro systems requirement (mainly power consumption), it is difficult to provide the gauge a large amount of heating current which is detrimental to its sensitivity. Jitchin \& Ludwig $^{3}$ have investigated the dynamical response of a macro-Pirani gauge to improve the sensor performance. However, since the heat diffusivity pattern alongside a micro-sized device is strongly different, their results cannot be directly applied. No other works have been reported on the transient behavior investigation of a micro-Pirani gauge. Transient time response analysis is an easy way to eliminate the non pressure dependent part of the gauge's signal when the gauge is heated by a current step. Advantageously the shape of the resulting signal (see Fig 2.) is very similar to the step response of a first order low-pass filter. Hence, post-processing using digital signal identification achieves an efficient way of performing pressure measurement.

\section{Results}

The non pressure dependent part of the transient signal is eliminated by subtracting the value of the first recorded point to the whole transient response. In post processing, the resulting signal (see Fig 2.) is identified to the step response of a low pass filter using digital Auto-Regressive Moving Average (ARMA) identification technique. The ARMA filter has been chosen of the lowest order possible. The gain of the obtained ARMA filter is taken as the measured value. The characteristic curves using the same experimental data are compared for the steady-state and the novel transient measurements (see Fig 3.). The sensitivity is enhanced by a factor of 16 with the later technique.

\footnotetext{
${ }^{1}$ K. Khosraviani, M. Leung, “The nanogap Pirani”, J. Micromech. Microeng. 19 (2009) 045007 (8pp).

${ }^{2}$ F.T. Zhang, "A micro-Pirani vacuum gauge based on micro-hotplate technology", Sensors and Actuators A 126 (2006) 300-305.

${ }^{3}$ W. Jitschin, S. Ludwig, "Dynamical behaviour of the Pirani sensor", Vacuum 75 (2004) 169-176.
} 
Figures

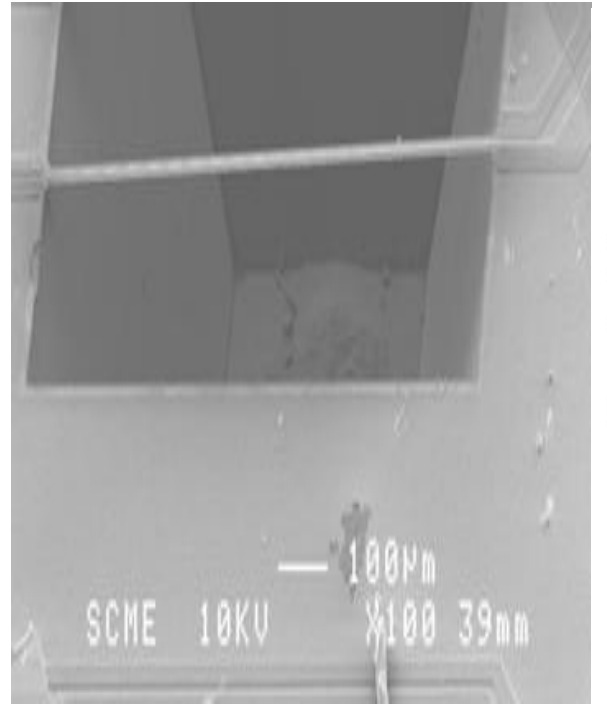

Fig. 1: A micro-Pirani gauge.

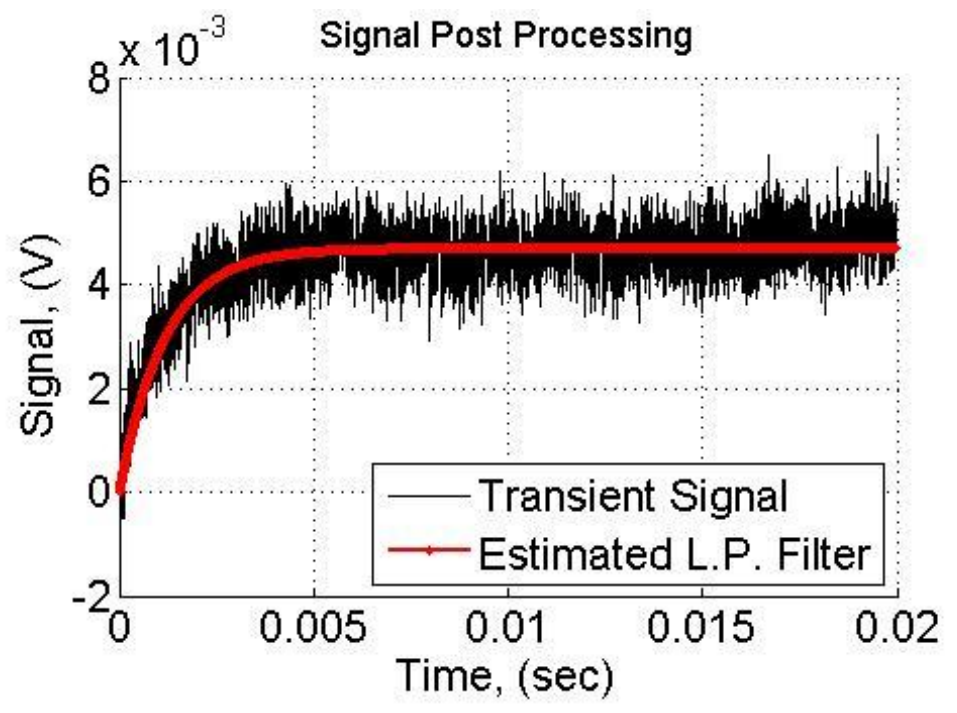

Fig. 2: Post-processing of the transient signal using identification with the step response of a Low Pass (L.P.) filter.

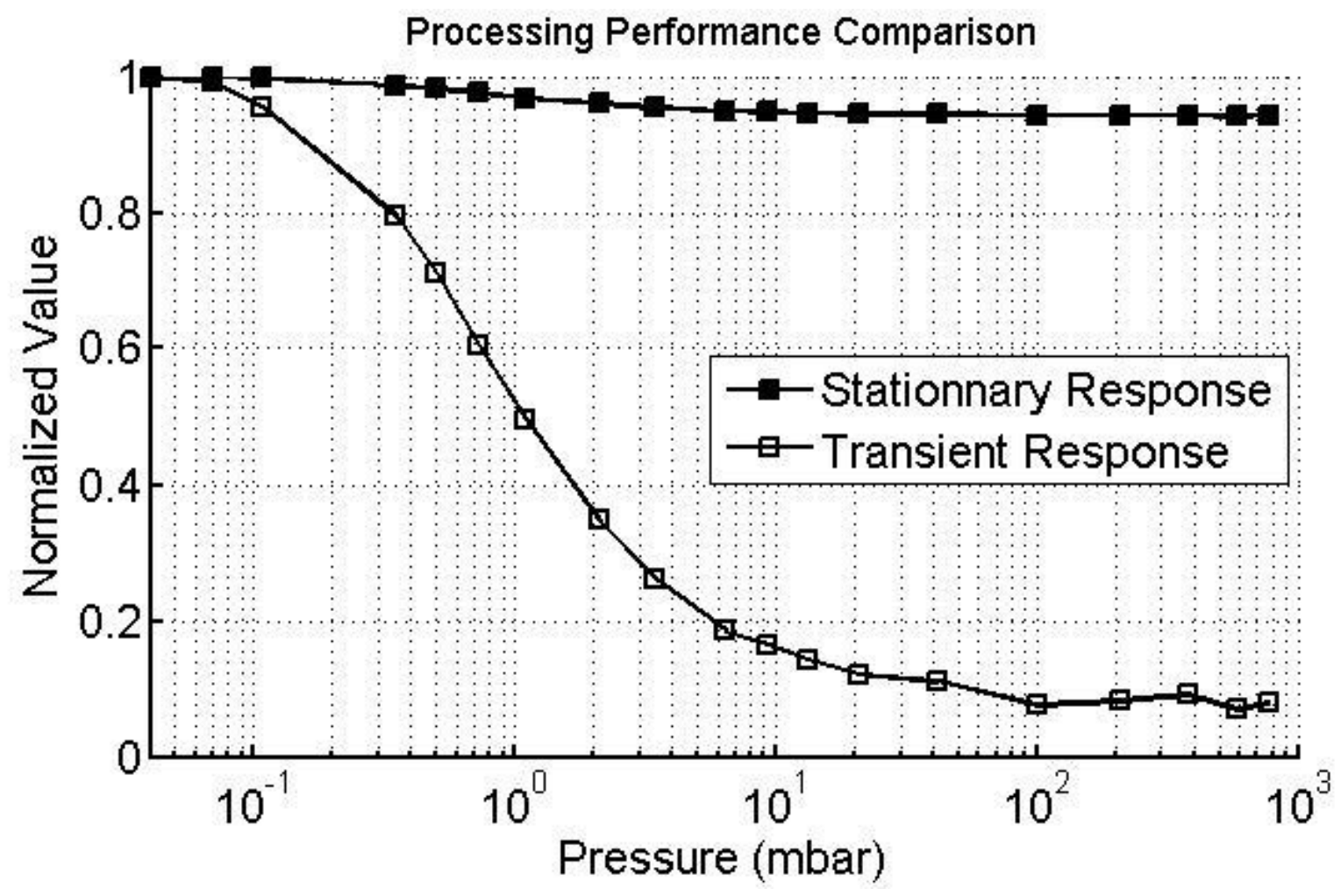

Fig. 3: Comparison of the pressure measurement for the stationary and the transient processing using experimental data. Vertical scale is normalized by the maximal value obtained for each type of processing. 